\title{
Lithium Toxicity at Therapeutic Blood Levels: A Case Report
}

\author{
Alexandra Quimby, BSc ${ }^{1}$, Rajeev Sachdeva, MD, $\operatorname{FRCP}(C)^{1,2}$
}

${ }^{1}$ Faculty of Medicine, University of Ottawa

${ }^{2}$ The Royal Ottawa Mental Health Centre

A BSTRACT

This report describes a case of lithium toxicity occurring at therapeutic blood levels in a 47-year-old man with bipolar disorder and a history of alcohol abuse. We discuss the clinical presentation of lithium toxicity, as well as factors that may contribute to a reduction in the blood levels required for the precipitation of toxic effects. In addition, we review the literature on cases of lithium toxicity occurring at therapeutic blood levels, including diagnosis and management thereof.

RÉ S U MÉ

Ce rapport décrit un cas de toxicité au lithium se produisant à des niveaux sanguins thérapeutiques chez un homme de 47 ans avec un trouble bipolaire et une histoire d'abus d'alcool. Nous discutons la présentation clinique de toxicité au lithium, ainsi que les facteurs pouvant contribuer à une réduction des niveaux sanguins nécessaires à la précipitation des effets toxiques. En outre, nous passons en revue la littérature sur les cas de toxicité au lithium se produisant à des niveaux sanguins thérapeutiques, y compris son diagnostic et sa gestion.

\section{INTRODUCTION}

Lithium is a first-line drug in the treatment of bipolar disorder, of which subtypes include bipolar I and bipolar II. Bipolar I is defined as the presence of at least one manic episode, though patients almost always also experience major depressive episodes. Bipolar II is defined as having suffered at least one hypomanic and at least one major depressive episode, as well as the absence of any manic episodes [1]. Lithium is indicated for the treatment of acute mania as well as maintenance therapy in bipolar disorder; treatment of acute bipolar depression is also an off-label use [2]. Historically, lithium's exact mechanism of action has been poorly understood [3]. However, recent evidence has shown lithium to play a neuroprotective role, increasing gray matter volumes in several brain areas central to the pathophysiology of mood disorders, including the amygdala, hippocampus, and prefrontal cortex. At the cellular level, lithium exerts its effects by decreasing excitatory and increasing inhibitory neurotransmission [4]. While it is an effective drug for the treatment of bipolar disorder, lithium is associated with GI, cardiac, and central nervous system (CNS) toxicity when blood levels exceed its narrow therapeutic window (0.6-1.2 mmol/L) [5]. Lithium toxicity may be of an acute or chronic nature. Acute toxicity occurs in individuals not being treated with lithium, typically in the setting of accidental or intentional overdose. Early symptoms of acute toxicity include nausea, vomiting, diarrhea, and ECG changes. With increasing doses, neurologic findings such as sluggishness, ataxia, confusion and neuromuscular excitability may develop as the drug is allowed to penetrate the CNS. Chronic toxicity occurs in individuals being treated with lithium who develop renal dysfunction. In the setting of renal dysfunction, individuals experience decreased lithium clearance, and thus increased serum lithium concentrations. Chronic toxicity often manifests as gradual development of neurologic symptoms similar to those of the late phase of acute toxicity. Treatment of lithium toxicity includes hydration and supportive care, discontinuation of lithium, and in cases of severe toxicity, hemodialysis. Even after discontinuation of lithium, long-term sequelae can persist and may include cerebellar dysfunction, extrapyramidal symptoms, brainstem dysfunction and dementia. These sequelae are collectively referred to as SILENT (syndrome of irreversible lithium effectuated neurotoxicity) [6-11].

\section{CASE REPORT}

\section{CASE PRESENTATION}

A forty-seven year old male with a history of bipolar II disorder and alcohol abuse was referred to The Royal Ottawa Mental Health Centre (ROMHC) Inpatient Mood Unit for assessment. The patient had an approximately two-month history of increasing depression, poor concentration and memory, and, at times, dysarthria and gait ataxia. He had a history of alcohol abuse, and had sustained two DUI charges in the past several months. Several months prior, he had been admitted to hospital for the treatment of a major depressive episode. His medications at the time

Keywords: Bipolar; Lithium toxicity; Pharmacotherapy 
included lithium and lamotrigine. He had been referred to ROM$\mathrm{HC}$ by his outpatient psychiatrist, who had performed a dementia workup to rule-out cognitive impairment, placed a neurology consultation, and ordered a CT and subsequent MRI head. Both of these imaging studies were reported as normal; neuropsychology testing was pending at the time of presentation. The working diagnosis at the time was psychomotor retardation as a feature of a major depressive episode. The patient was admitted to the Mood Unit for monitoring and further assessment.

\section{DIAGNOSIS \& TREATMENT}

Once admitted to hospital, the patient was treated with three doses of intramuscular thiamine due to the possibility of Wernicke's encephalopathy. Blood work was drawn including a complete blood count, B12, ferritin, folate, TSH, liver enzymes, and lithium levels. All blood work was reported within normal limits; the patient's serum lithium level was at the lower end of the therapeutic range, at $0.62 \mathrm{mmol} / \mathrm{L}$. Over the course of a week, the patient's mood gradually stabilized and returned to baseline. However, daily progress assessments revealed a lack of resolution of the patient's ataxia and dysarthria. The patient also began to report diplopia shortly after admission, which persisted thereafter. Tapering of lamotrigine was then attempted. Lamotrigine is an anticonvulsant drug shown to be efficacious as prophylaxis for bipolar depression. However, lamotrigine does not have a well-established therapeutic range for the treatment of bipolar disorder, and has the potential to cause adverse reactions similar to those experienced by the patient $[12,13]$. No change in the patient's symptoms occurred following tapering of lamotrigine. After several weeks of persistent symptoms, the patient himself broached the possibility of lithium toxicity occurring at therapeutic blood levels. The patient was reassured that this was extremely unlikely, but given his lack of symptom resolution, a decision was made to trial weaning from lithium. The patient's lithium dose was thus decreased in a step-wise fashion over a three-day period and finally stopped completely. On the fourth day, no symptom improvement had been noted, so the patient was transported to The Ottawa Hospital - Civic Campus for assessment by neurology. A repeat $\mathrm{CT}$ head with contrast was done, and the findings and assessment of the attending neurologist were threefold. Firstly, it was noted that there was a moderate degree of cerebellar vermal atrophy. Though impossible to ascertain a definitive cause for this finding, it was felt to likely be a result of chronic alcohol consumption. Secondly, the patient's symptoms of ataxia and speech incoordination were felt to be symptoms of lithium toxicity, the threshold for which had been lowered as a result of the underlying organic neurological impairment. Thirdly, the patient was found to have decreased visual acuity in the right eye, explaining his symptom of diplopia. The patient was sent back to ROMHC.

\section{OUTCOME}

Subsequently, on the morning of day five since beginning lithium tapering, the patient began to show improvement in both his speech and gait. By the evening of the same day, the patient's gait and speech had retuned to baseline. These findings were in keeping with the pharmacokinetic principle of complete drug clearance occurring after five half-lives: for lithium, the half-life is on average 24 hours. The decision was made for the patient to remain off of lithium, and to use lamotrigine instead as a mood stabilizer. Lamotrigine was subsequently titrated to therapeutic levels over the course of several weeks, to a final therapeutic dose of $200 \mathrm{mg} /$ day in two divided doses. The patient's mood remained stable, and he was discharged from hospital.

\section{DISCUSSION}

Though rare, a few reports exist of patients who have exhibited findings of lithium toxicity at normal serum lithium levels [14-18]. In one such report by Bell et al. (1993), four cases are described, and it is outlined that risk factors for developing lithium toxicity at therapeutic levels include pre-existing EEG changes, undetected cerebral pathology, and pre-existing organic impairment, as well as rapid dose increases and genetic susceptibility. For our patient, it was proposed that the observed cerebellar vermal atrophy, which was likely secondary to the effects of long-term high alcohol consumption, had acted as a "primer" which allowed the cerebellar symptoms of lithium toxicity to develop at lower blood levels than what would normally be considered toxic. It was thus the interaction of lithium with pre-existing cerebellar pathology that precipitated the patient's toxic symptoms, despite lithium levels at the lower end of the therapeutic range. In keeping with our case, it has been noted in the literature that serum lithium levels may be misleading. Clinical symptoms of toxicity may correlate more accurately with red blood cell lithium levels, which better reflect intracellular drug accumulation [16]. Thus, our case has shown that clinical manifestations, rather than serum lithium levels, should be the primary basis for diagnosis and treatment of lithium toxicity.

\section{REFERENCES}

1. American Psychiatric Association. Diagnostic and Statistical manual of Mental Disorders, Fifth Edition (DSM-5), American Psychiatric Association, Arlington, VA 2013.

2. American Psychiatric Association. Practice guidelines for the treatment of patients with bipolar disorder. American Journal of Psychiatry 2002;159(4 Suppl):1-50.

3. Singer I, Rotenberg D. Mechanisms of lithium action. N Engl J Med 1972;289:254.

4. Malhi GS, Tanious M, Das P, Coulston CM, Berk M. Potential mechanisms of action of lithium in bipolar disorder. CNS Drugs 2013;27:135-153.

5. Timmer RT, Sands JM. Lithium intoxication. J Am Soc Nephrol 1999;10:666.

6. Amisden A. Clinical features and management of lithium poisoning. Med Toxicol Adverse Drug Exp 1988;3:18.

7. Hansen HE, Amidisen A. Lithium intoxication. (Report of 23 cases and review of 100 cases from the literature). Q J Med 1978;47:123. 


\section{Case Report}

8. Okusa MD, Crystal LJ. Clinical manifestations and management of acute lithium intoxication. Am J Med 1994;97:383.

9. Hauger RL, O'Connor KA, Yudofsky S, Meltzer HL. Lithium toxicity: when is hemodialysis necessary? Acta Psychiatr Scand 1990;81:515.

10. Adityanjee, Munshi KR, Thampy A. The syndrome of irreversible lithiumeffectuated neuroxicity. Clin Neuropharmacol 2005;28(1):38.

11. Perrone J, Chatterjee P. Lithium Poisoning. In: UpToDate, Post TW (Ed), UpToDate, Waltham, MA. (Accessed on May 8, 2015.)

12. Ng F, Hallam K, Lucas N, Berk M. The role of lamotrigine in the management of bipolar disorder. Neuropsychiatr Dis Treat 2007;3(4):463-474.

13. Lexi-Comp, Inc. (Lexi-Drugs ${ }^{\circledR}$ ). Lexi-Comp, Inc.; August 11, 2015.

14. Bell, A J ; Cole, A ; Eccleston, D ; Ferrier, I N. Lithium neurotoxicity at normal therapeutic levels. Br J of Psychiatry 1993;162:689-692.

15. Speirs J, HIrsch SR. Severe lithium toxicity with "normal" serum concentrations. Br Med J. 1978;1(6116):815.

16. Venkatarathnamma PN, Patil AR, Nanjundaiah N. Fatal lithium toxicity with therapeutic levels- a case report. Int J Clin Pharmacol Ther. 2011 May;49(5):336-8.

17. Miao YK. Lithium neurotoxicity within the therapeutic serum range.(Case Report). Hong Kong Journal of Psychiatry, March, 2002, Vol.12(1), p.19(4)

18. Gangadhar BN, Subhash MN, Umapathy C, Janakiramaiah N. Lithium toxicity at therapeutic serum levels. The British Journal of psychiatry 1993;163:695. 